\title{
Prevalencia de labio ylo paladar fisurado y factores de riesgo
}

Sacsaquispe S, Ortiz L. Prevalencia de labio y/o paladar fisurado y factores de riesgo. Rev Estomatol Herediana 2004;14(1-2) : 54 - 58.

\section{RESUMEN}

El Labio y Paladar Fisurado (LPF) es una malformación bucomaxilar congénita frecuente. El objetivo de este trabajo fue establecer la prevalencia de LPF en el Instituto Especializado Materno Perinatal y su relación con los factores de riesgo, en los años 2001 y 2002. Se revisaron las historias clínicas de neonatos con LPF y de sus madres. Se utilizó la clasificación de fisuras orales propuestas por Kernaham y Stark. De 37,213 nacimientos se hallaron 44 casos encontrándose una prevalencia de 1 por mil nacimientos. La fisura labio palatina se presentó con mayor frecuencia (68\%). No se encontró ninguna relación estadísticamente significativa con respecto a la edad materna. La frecuencia fue mayor en el sexo masculino $(54.55 \%)$ que en el sexo femenino (45.5\%). Se encontró asociado a otra malformación en el $40.9 \%$. El 31.7\% presentó antecedentes de consumo de medicamentos durante el primer trimestre. Se encontró una diferencia estadísticamente significativa en relación a la presencia de enfermedad en el embarazo y el LPF. En conclusión es una patología de etiología multifactorial.

Palabras clave: LABIO LEPORINO / PALADAR FISURADO.

\section{Prevalence of cleft lip and/or palate and risk factors}

ABSTRACT

Cleft lip and palate (CLP) is a frequent congenital malformation of the mouth. The aim of this study was to establish the prevalence of CLP and its relationship with risk factors. Clinical histories of newborns and of their mothers were reviewed. CLP were recorded according to Kernaham and Stark (1958) oral fissures classification. Of 37,213 births, 44 cases were found, the prevalence being of 1:1000 in which the cleft lip with cleft palate was present with a higher frequency (68\%), statistical significant relation with regard to maternal age was not found. Frequency was higher in males (54.55\%) than in females (45.5\%). Association with another malformation was of $40.9 \%$. The mothers of $31.7 \%$ had medicine intake significant the first quarter of pregnancy. A statistical significant difference with regard to CLP and illness during pregnancy was found. The conclusion was established that the etiology of CLP is due to multiple factors.

Keywords: CLEFT LIP / CLEFT PALATE.
Sonia Sacsaquispe Contreras ${ }^{1}$ Luz Ortiz ${ }^{2}$

'Docente del Departamento Académico de Medicina Cirugía y Patología Oral.

Facultad de Estomatología. Universidad Peruana Cayetano Heredia.

${ }^{2}$ Cirujano Dentista.

\section{Introducción}

Las fisuras labio palatinas constituyen deficiencias estructurales congénitas debidas a la falta de unión entre algunos procesos faciales embrionarios en formación. Las fisuras del paladar primario y las fisuras raras de la cara, las cuales incluyen los procesos faciales, ocurren en el período embrionario, a partir de la sexta semana de vida intrauterina, mientras que las fisuras del paladar secundario ocurren a partir de la octava semana de vida prenatal, ya en el periodo fetal (1). Hay evidencia que una predisposición hereditaria junto a la conjugación de factores ambientales puede precipitar la aparición de esta anomalía. Aproximadamente un $30 \%$ de los casos se debe a factores hereditarios (2). Se ha encontrado que tanto el labio figurado como la fisura de labio y paladar son más frecuentes en el sexo masculino mientras que el paladar fisurado era más frecuente en el sexo femenino. Entre un 50\% a 70 \% de las fisuras de labio y/o paladar corresponden a formas no sindrómicas, a más de 300 entidades reconocibles (3). La prevalencia de las fisuras orales han sido relacionadas con el número de gestaciones de la madre, siendo más prevalentes en los casos donde la madre es multípara $(4,5)$, y en relación a la edad materna Aizpurua (4) registró que la mayor frecuencia se daba en madres de 26 a 30 años (50\%); por otra parte Lofiego (6) encontró que el mayor número de casos se daba entre las madres que eran mayores de 40 años. Esta anomalía del desarrollo no ha sido muy estudiada en nuestro país, hay pocos estudios de labio y/o paladar figurado y su relación con los factores de riesgos asociados; es por ello que se consideró conveniente realizar este estudio para establecer su prevalencia actual y establecer los factores que pudieran estar asociados.

\section{Material y método}

Se revisaron las historias clínicas de los neonatos nacidos en los años 2001 y 2002 en el Instituto Especializado Materno Perinatal que presentaron labio y/ o paladar fisurado y de sus madres. Se analizaron las siguientes variables: - Tipo de fisuras de acuerdo a la cla- 
sificación de Kernaham y Stark (7) (Fig. 1,2 y 3) :

Grupo I : Hendiduras del paladar primario: (labio y premaxila).

1.- Unilateral : (total y sub-total)

2.- Mediana : (total y sub-total)

3.-Bilateral : (total y sub-total)

Grupo II : Hendiduras del paladar secundario : (total, sub-total y submucoso).

Grupo III : Hendiduras de paladar primario y secundario

1.- Unilateral : (total y sub-total)

2.- Mediana : (total y sub-total)

3.-Bilateral : (total y sub-total)

- Sexo del neonato.

- Número de gestaciones de la madre: Se dividió en tres grupos : de una sola gestación, de dos gestaciones y de tres a más gestaciones.

- Edad de la madre : Se dividió en 4 grupos: menores de 20 años, de 20 a 25, 26 a 30 y mayores de 30 años.

- Antecedente familiar: variable cualitativa que determinó la relación de los antecedentes familiares.

- Asociación a otra malformación: variable cualitativa que determinó la presencia de otra malformación congénita.

- Consumo de medicamentos durante el primer trimestre de embarazo.

- Enfermedades durante el embarazo. Se utilizarón las historias que tuvieron los datos completos.

Se tomaron los datos de las historias clínicas de los neonatos y de sus madres, los cuales fueron registrados en fichas confeccionadas especialmente para el estudio.

Análisis estadístico: Los datos recolectados de la ficha diseñada para la investigación fueron tabulados, obteniéndose así distribución de frecuencias y porcentajes.

Se realizó el análisis descriptivo de los datos utilizando tablas, gráficas de frecuencia de labio y/o paladar fisurado según la variable.

Para el ingreso de información de la base de datos se utilizó el programa Excel y para el correspondiente análisis se utilizó el programa estadístico SPSS v.10.

\section{Resultados}

Se encontraron 44 casos de labio y/ o paladar fisurado en un total de 37,213 neonatos registrados en los años 2001 y 2002 dando una prevalencia de 0.001 . El 55\% de los casos correspondían al sexo masculino. El 68.18\% corresponden al grupo III, el 25\% al II y el 6.2\% al I (Tabla 1)

El mayor número de casos (40.9\%) de fisuras correspondió al grupo de madres cuyo número de gestaciones fue igual o mayor a tres niños. No se encontró una diferencia estadísticamente significativa con respecto a la edad de la madre.

En relación a la presencia de antecedentes familiares se encontró que sólo el $11.4 \%$ lo tuvo siendo el grupo III el más asociado; el grupo I no tuvo an- tecedentes familiares.

El 40.9\% de los neonatos presentó otra malformación asociada como : alteraciones de orejas y nariz, Trisomia 13, T. 21 y Holoprosencefalia; siendo el grupo III el más relacionado (Fig. 4).

Se encontró que el $31.7 \%$ de las madres consumió algún medicamento durante el primer trimestre de embarazo tales como amoxicilina (20\%), nitrato de miconazol (13.3\%), dimenhidrinato (13.3\%), fenitoina, betametasona, ampicilina, cefalosporinas, metronidazol, tetraciclinas en menores porcentajes. (Tabla 2)

En relación a la presencia de enfermedad de la madre durante el embarazo se observó que en el total de los casos el 50\% de las madres tuvo alguna enfermedad durante el periodo de gestación (Fig. 5), tales como : anemia (35\%), preeclampsia (20\%), pre-eclampsia más anemia (10\%), infección del tracto urinario (10\%), epilepsia, enfermedad de transmisión sexual, disfunción renal en menores porcentajes. Los grupos II y III fueron los más relacionados.

\section{Discusión}

En el presente estudio se encontró una prevalencia de un caso por cada mil nacimientos; esta prevalencia es relativamente baja en comparación con la población asiática, chilena y estadounidense donde la relación es de 1/450, 1 / 500 y 1 por cada 700 recién nacidos vivos respectivamente (6), estudios simi-

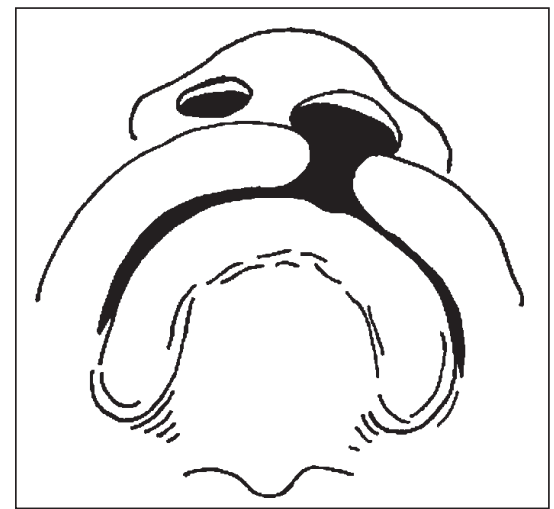

Fig.1. Hendidura paladar primario (tomado de Bhaskar SN. Histología y embriología bucal de Orban. 11va. Ed. Mexico DF:Pardo, 1993).

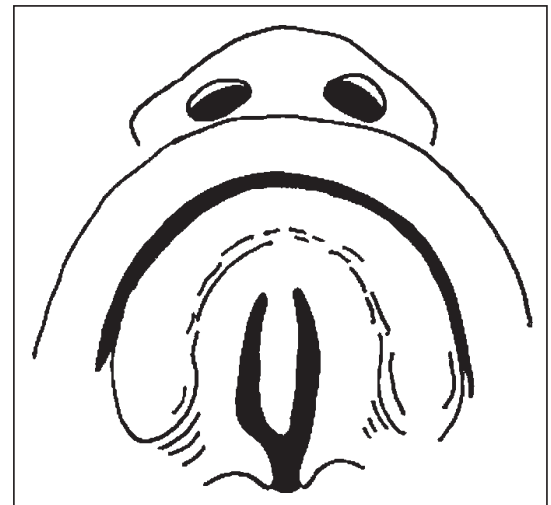

Fig. 2. Hendidura paladar secundario (tomado de Bhaskar SN. Histología y embriología bucal de Orban. 11va. Ed. Mexico DF:Pardo, 1993).

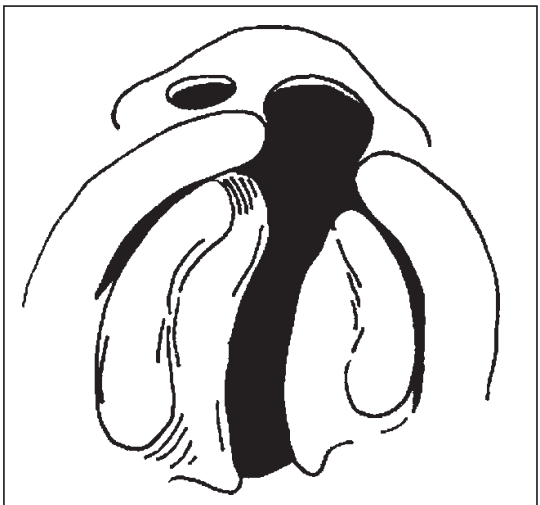

Fig. 3. Hendidura paladar primario y secundario (tomado de Bhaskar SN. Histología y embriología bucal de Orban. 11va. Ed. Mexico DF:Pardo, 1993). 
Tabla 1. Neonatos con labio y paladar fisurado

\begin{tabular}{|c|c|c|c|c|c|c|}
\hline \multirow{3}{*}{$\begin{array}{l}\text { DX } \\
\text { GRUPO }\end{array}$} & \multicolumn{4}{|c|}{ SEXO } & \multirow{2}{*}{\multicolumn{2}{|c|}{ Total }} \\
\hline & \multicolumn{2}{|c|}{ Masculino } & \multicolumn{2}{|c|}{ Femenino } & & \\
\hline & $\mathbf{n}$ & $\%$ & $\mathbf{n}$ & $\%$ & $\mathbf{n}$ & $\%$ \\
\hline I & 1 & 2.27 & 2 & 4.55 & 3 & 6.82 \\
\hline II & 6 & 13.64 & 5 & 11.36 & 11 & 25 \\
\hline III & 17 & 38.64 & 13 & 29.55 & 30 & 68.18 \\
\hline Total & 24 & 54.55 & 20 & 45.45 & 44 & 100 \\
\hline
\end{tabular}

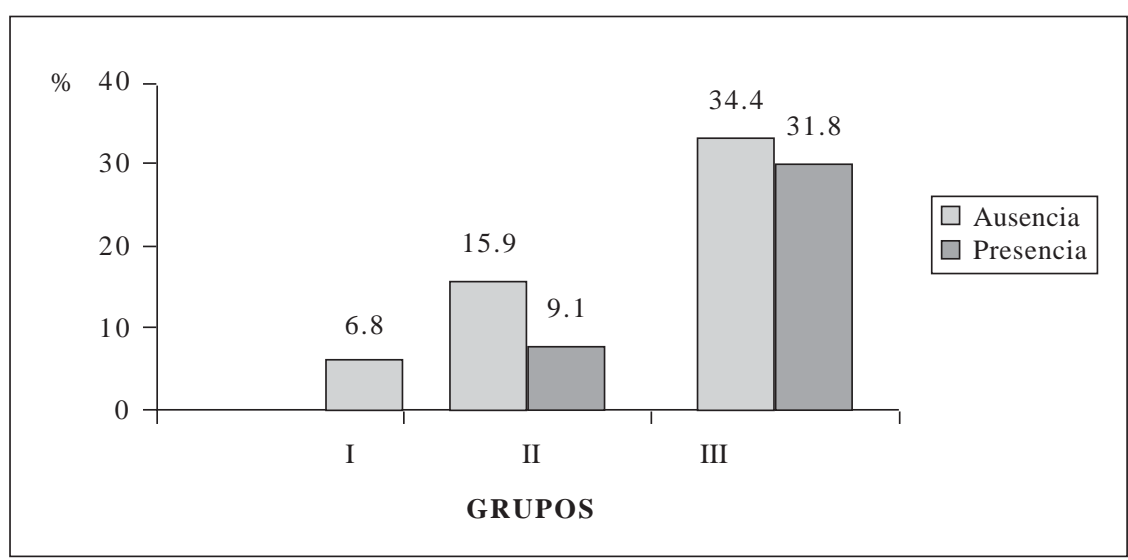

Fig. 4. Neonatos con labio y paladar fisurado según grupo y presencia o ausencia de otra malformación

Tabla 2. Neonatos con labio y paladar fisurado según grupo y consumo de medicamentos.

\begin{tabular}{|c|c|c|c|c|c|c|}
\hline \multirow{3}{*}{$\begin{array}{l}\text { DX } \\
\text { GRUPO }\end{array}$} & \multicolumn{4}{|c|}{ Consumo de Medicamentos } & \multirow{2}{*}{\multicolumn{2}{|c|}{ Total }} \\
\hline & NO & & SI & & & \\
\hline & $\mathbf{n}$ & $\%$ & $\mathbf{n}$ & $\%$ & $\mathbf{n}$ & $\%$ \\
\hline I & 1 & 2.4 & 2 & 4.9 & 3 & 7.3 \\
\hline II & 6 & 14.6 & 3 & 7.3 & 9 & 22 \\
\hline III & 21 & 51.2 & 8 & 19.5 & 29 & 70.7 \\
\hline Total & 28 & 68.3 & 13 & 31.7 & 41 & 100 \\
\hline
\end{tabular}

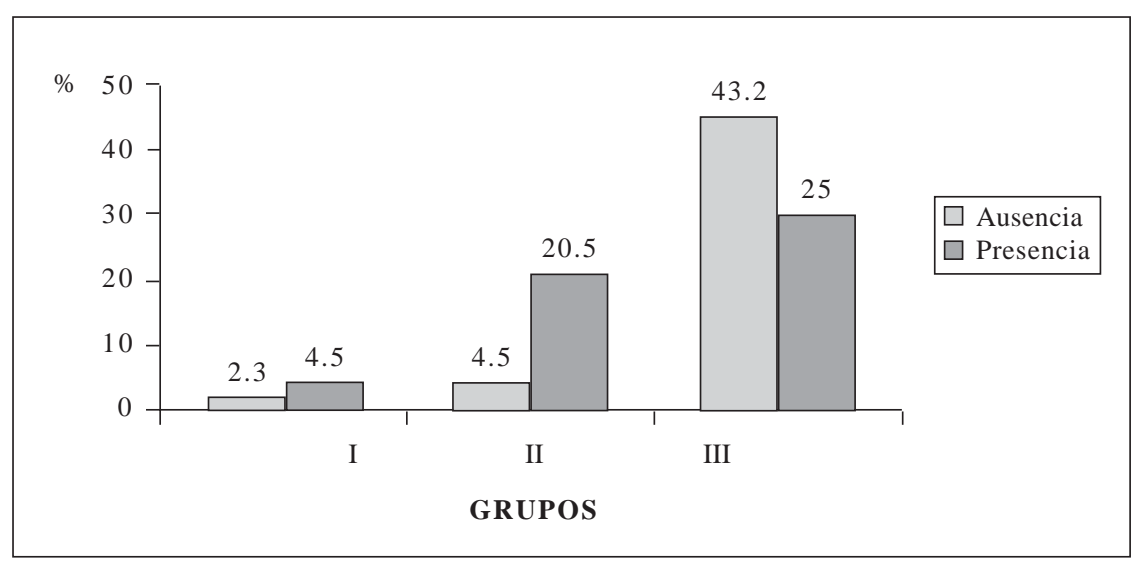

Fig. 5. Neonatos con labio y paladar fisurado según grupo y presencia o ausencia de enfermedad en el embarazo. lares en nuestro país como los de Aleman (5), Arcaya (8), Klein (9) hallaron una prevalencia de 0.96 -1.2 por cada mil nacimientos que es similar a lo reportado en este estudio.

Los resultados del estudio muestran que el labio y paladar fisurado y la fisura del paladar aislado son más frecuentes en el sexo masculino, mientras que el labio fisurado aislado es más frecuente en el sexo femenino; lo que difiere de lo hallado por Cedeño et al. (10) que encontraron que el labio fisurado es más común en el sexo masculino y que el paladar fisurado aislado es más común para el sexo femenino. Por otra parte, Arcaya (8) en nuestro país encontró que el grupo labio y paladar fisurado es más común en el sexo masculino y que las fisuras palatinas afectan más al sexo femenino.

Con respecto a la presencia de otra malformación se encontró que el 40.9\% de las fisuras orales están asociadas a otra malformación congénita, lo cual es un porcentaje alto comparándolo con lo registrado por Longhi (13.4\%), Lorentz, Westmorelan y Richars (18.2\%), Hodswotan (20\%) y Calzolari (33\%) (12). El grupo de labio y paladar fisurado (Grupo III) es el que esta más relacionado con la presencia de otra malformación, lo que difiere de Longhi (11) quien encontró que el grupo de fisura palatina aislada era el más afectado.

En cuanto a la variable de edad materna los grupos que obtuvieron mayor frecuencia fueron el 20 a 25 años y el de mayores de 30 años con 34.1\% y 31.8\% respectivamente, al igual que Aleman (5) no se encontró ninguna relación estadísticamente significativa entre la ocurrencia de fisuras orales y la edad materna.

Con relación al número de gestaciones se encontró que el mayor porcentaje de fisuras ocurre en el grupo de $3 \mathrm{a}$ más gestaciones, lo cual coincide con lo encontrado por Aizpurua (5) quien observó que la mayoría de los recién nacidos con fisura labiopalatina corresponden a madres multíparas.

Con respecto a la presencia de antecedentes familiares el $11.4 \%$ de todos los casos presentaron antecedentes familiares, en otros estudios han determi- 
nado que es un factor de riesgo importante tanto para el labio fisurado como para el paladar fisurado (13), con riesgos relativos de 4.96 y 2.58 respectivamente. En el estudio realizado por Muñoz, Bustos et al (14) considerando conjuntamente las dos anomalías, el riesgo fué de 2.14. Klein (9) registró que el $22 \%$ de los casos con fisuras orales presentaron antecedentes familiares. En general la causa es desconocida en el $40 \%$ al 60\% de las anomalías congénitas.

Con respecto a la presencia de otra malformación se encontró que el 40.9\% de las fisuras orales están asociadas a otra malformación congénita, lo cual es un porcentaje alto comparándolo con lo registrado por Longhi (13.4\%), Lorente, Westmorelan y Richard (18.2\%), Hodswotan (20\%) y Calzolari (33\%) (12). El grupo de labio y paladar fisurado (grupo III) es el que esta más relacionado con la presencia de otra malformación, lo que difiere de Longhi (11) quien encontró que el grupo de fisura palatina aislada era el más afectado. En otros estudios se encontró que el labio y/o paladar fisurado se presentan en un $70 \%$ no sindrómicas y en un $30 \%$ asociadas a otra malformaciones. Las asociaciones son más frecuentes en los pacientes con paladar fisurado aislado (61\%). Existen aproximadamente 350 sindromes que presentan labio y/o paladar fisurado (13).

El 31.7\% de las madres consumieron algún medicamento durante el primer trimestre de embarazo y los más comunes fueron: amoxicilina, nitrato de miconazol y dimenhidrinato. Los estudios preclínicos muestran que la amoxicilina carece de efectos teratogénicos, no se recomienda el uso de nitrato de miconazol en el embarazo sobre todo en el primer trimestre pues no ha sido estudiado del todo su seguridad y no se ha encontrado contraindicaciones para el dimenhidrinato (14). Otros estudios han reportado que el diazepan y las benzodiazepinas actúan como teratógenos en la formación de fisuras bucales; los esteroides y los anticonvulsivos también están relacionados y las anfentaminas demostraron causar fisuras bucales y otras condiciones anómalas (11). Muñoz et al. (13) indicaron que el riesgo de tener un niño con labio y/o paladar fisurado cuando se consume medicamentos durante la gestación es cinco veces el riesgo que cuando no se consumen y dos veces cuando hay antecedentes de malformaciones familiares que cuando no los hay. Hay pocos fármacos teratógenos para los que se haya demostrado una relación causa-efecto. La teratogénesis espontánea se sitúa alrededor del 4.4\% de los nacidos (15). El período durante el que un fármaco tiene mayor riesgo de producir alteraciones congénitas es durante las primeras ochos semanas de embarazo (período de organogénesis), pero la posibilidad de producir efectos adversos persiste a lo largo de toda la gestación (16).

En cuanto a la relación de enfermedad materna en el embarazo se encontró que el 50\% de las madres sufrieron alguna enfermedad en el embarazo, siendo la anemia la más común, seguida por la pre-eclampsia, amenaza de aborto, infecciones intrauterinas; aunque la literatura se registra que la mayoría de las causas ambientales son infecciones de la madre (que llegan a hacer el $2 \%$ de las causas de las anomalías congénitas) otras afecciones maternas como diabetes, fenilcetonuria, enfermedades virales también están relacionadas (10). La anemia es la complicación hematológica más frecuente durante el embarazo, parto y puerperio. El hierro es fundamental para prevenir la anemia, que durante el embarazo retarda el crecimiento del feto, aumenta la posibilidad de la madre y del niño en un $2.5 \%$, acrecienta la posibilidad de pérdida del bebe, las malformaciones genéticas, y el bajo peso del recién nacido; también produce un aumento de la frecuencia de partos prematuros y deformidades en el sistema nervioso del feto.

En conclusión al estudiar el labio y/ o paladar fisurado y sus factores de riesgo se encontraron algunos factores que nos han sido reportado en otras investigaciones como es el consumo de amoxicilina, nitrato de miconazol y dimenhidrinato en el primer trimestre de embarazo y el incremento en la relación de otras malformaciones congénitas vinculadas al labio y/o paladar fisurado, lo que contribuye a conocer mejor esta patología de carácter congénito.

\section{Referencias bibliográficas}

1. Ruiz IR. Embriología facial: Diagnóstico de las Principales Malformaciones Congénitas. [Sitio en Internet] Secpre. Disponible en : URL :

http://www.usuarios.tiscali.es/ pacod/afilapa/fisura.htm .

2. Franklin E. Fisura Oral y/o Facial. [Sitio en Internet] Marcha of Dimes Birth Defects Fundation. Disponible en : URL :

http://www.nacersano.org/Bib/

3. Zarante I, Castillo M. Análisis clínico epidemiológico de factores asociados a malformaciones congénitas ECLAMC- Hospital Universitario San Ignacio Junio-Diciembre del 2001. [sitio en Internet] Pontificia Universidad Javeriana. Disponible en : URL :

http://med.javeriana.edu.co/publi/ v n i v e r s i t a s / v 43 n 2 / 0031\%20ANALISIS.pdf

4. Aizpurua R. Incidencia de Labio Leporino y Paladar Hendido en una Región de Venezuela. Rev Lat Orto Odontopd [publicación periódica en línea] 2002. Agosto [citada 2003 Ene 25] Se encuentra en : URL :

http://www.ortodoncia.ws/

5. Aleman M. Incidencia de fisura labial, palatina y labiopalatina, en relación al sexo, rol de fratría y edad de la madre, en tres hospitales de Lima Metropolitana y uno del Callao durante una década (1972-1981). [Tesis para optar el Título de Cirujano Dentista]. Lima, Perú. Universidad Peruana Cayetano Heredia, Facultad de Estomatología, 1983.

6. Chaves F, Salete P, Dimare D, Jaeger C. Fisuras Labiopalatais : Aspecto Epidemiológicos e Etiologia-Revisao da Literatura. Rev Odonto Ciencia 1999;(28)2:121-28

7. Báez R, Bastidas R, Monserat E. Labio y paladar hendido. Act Odont Ven 2000;38(3): 15-20

8. Arcaya A. Frecuencia de Fisuras Labiales, Palatinas y Labiopalatinas 
en el Hospital Nacional Cayetano Heredia y en pacientes de la Clínica Estomatológica Central durante los años 1994-1999 [Tesis para optar el título de Cirujano Dentista]. Lima, Perú. Universidad Peruana Cayetano Heredia, Facultad de Estomatología, 2001

9. Klein E. Tesis Genética de Fisuras Labiales, Labiopalatinas y Fisuras Palatina. Tesis para optar el título de Cirujano General. Universidad Peruana Cayetano Heredia, Facultad de Medicina General,1974.

10. Cedeño J, Ghanem A, Maza W. Labio y Paladar hendidos. Reporte de un caso. Act Odont Ven 1999; 38(2): 56-60.

11. Longhi T. Frecuencia de Fisura La- bial, Palatina y Labiopalatina asociada a otras Malformaciones Congénitas y posibles Agentes Causales. [Tesis para optar el título de Cirujano Dentista]. Lima, Perú. Universidad Peruana Cayetano Heredia, Facultad de Estomatología, 1983.

12. Derickje A, Eerens A, Carel C. The incidence of Oral Clefts: a rewiew. Br J Oral Max Surg 1996;34: 488-96

13. Muñoz J, Bustos I, Quintero C, Giraldo A. Factores de Riesgo para Algunas Anomalías Congénitas en Población Colombiana. [sitio en Internet] Revista de Salud Publica. Disponible en: URL: http:// www.medicina.unal.edu.co/

14. Pannone R, Sosa L. Fármacos en el
Embarazo y la Lactancia.[sitio en Internet] Sitio Médico. Disponible en URL: http://www.sitiomedico.com

15. Serrano JS, Serrano MI y Rodríguez JN. Uso de medicamentos durante el embarazo. Medicine 1991 ; 92(5):3607-16.

16. García Arenillas M. Utilización de medicamentos en embarazo, lactancia, niños y ancianos. Medicine1999;30(2): 6158-6165.

\section{Dirección de correspondencia}

Sonia Sacsaquispe Contreras.

Calle 24 Nº 185 Urb. Corpac Lima 27 -

Perú.

9924-0648

Luz_yob@hotmail.com 